\title{
Differential superordination for harmonic complex-valued functions
}

\author{
Georgia Irina Oros and Gheorghe Oros
}

\begin{abstract}
Let $\Omega$ and $\Delta$ be any sets in $\mathbb{C}$, and let $\varphi(r, s, t ; z): \mathbb{C}^{3} \times U \rightarrow \mathbb{C}$. Let $p$ be a complex-valued harmonic function in the unit disc $U$ of the form $p(z)=p_{1}(z)+\overline{p_{2}(z)}$, where $p_{1}$ and $p_{2}$ are analytic in $U$. In [5] the authors have determined properties of the function $p$ such that $p$ satisfies the differential subordination

$$
\varphi\left(p(z), D p(z), D^{2} p(z) ; z\right) \subset \Omega \Rightarrow p(U) \subset \Delta .
$$
\end{abstract}

In this article, we consider the dual problem of determining properties of the function $p$, such that $p$ satisfies the second-order differential superordination

$$
\Omega \subset \varphi\left(p(z), D p(z), D^{2} p(z) ; z\right) \Rightarrow \Delta \subset p(U) .
$$

Mathematics Subject Classification (2010): 30C80, 30C46, 30A20, 34A40.

Keywords: Differential subordination, harmonic functions, differential superordination, subordinant, best subordinant, analytic function.

\section{Introduction and preliminaries}

The theory of differential subordinations (or the method of admissible functions) for analytic functions was introduced by S.S. Miller and P.T. Mocanu in papers [6] and [7] and later developed in [1], [8], [10], [11], [12], [13].

The theory of differential subordinations has been extended from the analytic functions to the harmonic complex-valued functions in papers [2], [5], [14].

Let $U=\{z \in \mathbb{C}:|z|<1\}$ be the open unit disc of the complex plane with

$$
\bar{U}=\{z \in \mathbb{C}:|z| \leq 1\} \text { and } \partial U=\{z \in \mathbb{C}:|z|=1\} .
$$

Denote by $\mathcal{H}(U)$ the class of holomorphic functions in the unit disc $U$, and

$$
A_{n}=\left\{f \in \mathcal{H}(U): f(z)=z+a_{n+1} z^{n+1}+\ldots\right\}, A_{1}=A .
$$


A harmonic complex-valued mapping of the simply connected region $\Omega$ is a complex-valued function of the form

$$
f(z)=h(z)+\overline{g(z)}
$$

where $h$ and $g$ are analytic in $\Omega$, with $g\left(z_{0}\right)=0$ for some prescribed point $z_{0} \in \Omega$.

We call $h$ and $g$ analytic and co-analytic parts of $f$, respectively. If $f$ is (locally) injective, then $f$ is called (locally) univalent. The Jacobian and the second complex dilatation of $f$ are given by

$$
J_{f}(z)=\left|h^{\prime}(z)\right|^{2}-\left|g^{\prime}(z)\right|^{2}
$$

and

$$
w(z)=g^{\prime}(z) / h^{\prime}(z), z \in \Omega, \text { respectively. }
$$

A function $f \in C^{2}(\Omega), f(z)=u(z)+i v(z)$ which satisfies

$$
\Delta f=\frac{\partial^{2} f}{\partial x^{2}}+\frac{\partial^{2} f}{\partial y^{2}}=0
$$

or

and

$$
\Delta u=\frac{\partial^{2} u}{\partial x^{2}}+\frac{\partial^{2} u}{\partial y^{2}}=0
$$

is called harmonic function.

$$
\Delta v=\frac{\partial^{2} v}{\partial x^{2}}+\frac{\partial^{2} v}{\partial y^{2}}=0
$$

By $\operatorname{Har}(U)$ we denote the class of complex-valued, sense-preserving harmonic mappings in $U$. We note that each $f$ of the form (1.1) is uniquely determined by coefficients of the power series expansion [2]

$$
h(z)=a_{0}+\sum_{n=1}^{\infty} a_{n} z^{n}, g(z)=b_{0}+\sum_{n=1}^{\infty} b_{n} z^{n}, z \in U,
$$

where $a_{n} \in \mathbb{C}, n=0,1,2, \ldots$ and $b_{n} \in \mathbb{C}, n=0,1,2, \ldots$

Several fundamental informations about harmonic mappings in the plane can also be found in [3].

For $f \in \operatorname{Har}(U)$, let the differential operator $D$ be defined as follows

$$
D f=z \frac{\partial f}{\partial z}-\bar{z} \frac{\partial f}{\partial \bar{z}}=z h^{\prime}(z)-\overline{z g^{\prime}(z)},
$$

where $\frac{\partial f}{\partial z}$ and $\frac{\partial f}{\partial \bar{z}}$ are the formal derivatives of function $f$

$$
\frac{\partial f}{\partial z}=\frac{1}{2}\left(\frac{\partial f}{\partial x}-i \frac{\partial f}{\partial y}\right) \text { and } \frac{\partial f}{\partial \bar{z}}=\frac{1}{2}\left(\frac{\partial f}{\partial x}+i \frac{\partial f}{\partial y}\right) .
$$

The conditions (1.4) are satisfied for any function $f \in C^{1}(\Omega)$ not necessarily harmonic, nor analytic.

Moreover, we define $n$-th order differential operator by recurrence relation

$$
D^{2} f=D(D f)=D f+z^{2} h^{\prime \prime}-\overline{z^{2} g^{\prime \prime}}, D^{n} f=D\left(D^{n-1} f\right) .
$$

Remark 1.1. If $f \in \mathcal{H}(U)$ (i.e. $g(z)=0$ ) then $D f(z)=z f^{\prime}(z)$. 
Now we present several properties of the differential operator $D f$.

Proposition 1.1. It is easy to prove that if $f, g \in \operatorname{Har}(U)$, then the linear operator $D$ satisfies the usual rules of differential calculus:

a) $D(f \cdot g)=f D g+g D f$

b) $D\left(\frac{f}{g}\right)=\frac{g D f-f D g}{g^{2}}$

c) $D(f \circ g)=\frac{\partial f}{\partial g} \cdot D g+\frac{\partial f}{\partial \bar{g}} \cdot D \bar{g}$

d) $D \bar{f}=-\overline{D f}$

e) $D \operatorname{Re} f=i \operatorname{Im} D f$

f) $D \operatorname{Im} f=-i \operatorname{Re} D f$

g) $D|f|=i|f| \cdot \operatorname{Im} \frac{D f}{f}$

h) $D \arg f=-i \operatorname{Re} \frac{D f}{f}$

If $z=r e^{i \theta}$, then

a) $\frac{\partial f}{\partial \theta}=i D f, r \frac{\partial D f}{\partial r}=D^{2} f$

b) $\frac{\partial}{\partial \theta} \arg f=\operatorname{Re} \frac{D f}{f}=\operatorname{Re} \frac{z h^{\prime}(z)-\overline{z g^{\prime}(z)}}{h(z)+\overline{g(z)}},(f(z) \neq 0)$

c) $\frac{\partial|f|}{\partial \theta}=-|f| \cdot \operatorname{Im} \frac{D f}{f}(f(z) \neq 0)$

In order to prove the main results of this paper, we use the following definitions and lemmas:

Definition 1.1. (Definition 2.2, [5]) By $Q$ we denote the set of functions

$$
q(z)=q_{1}(z)+\overline{q_{2}(z)}
$$

harmonic complex-valued and univalent on $\bar{U} \backslash E(q)$, where

$$
E(q)=\left\{\zeta \in \partial U ; \lim _{z \rightarrow \zeta} f(z)=\infty\right\} .
$$

Moreover, we assume that $D(q(\zeta)) \neq 0$, for $\zeta \in \partial U \backslash E(q)$. The set $E(q)$ is called an exception set. We note that the functions

$$
q(z)=\bar{z}, q(z)=\frac{1+\bar{z}}{1-\bar{z}}
$$

are in $Q$, therefore $Q$ is a non-empty set.

For the number $0<r<1$, we denote by $U_{r}=\{z \in \mathbb{C}:|z|<r\}$. 
Lemma 1.1. (Lemma $2.2[5])$ Let $p, q \in \operatorname{Har}(U), p(U)$ be simply connected and $q$ be univalent in $U$. Also, let $p \in Q$ with $p(0)=q(0)=1, q(z) \neq 1$. If $q$ is not strongly subordinate to $p$, then there exist points $z_{0}=r_{0} e^{i \theta_{0}}$ and $\zeta_{0} \in \partial U \backslash E(q)$ and a number $m \geq 1$ such that $q\left(U_{r_{0}}\right) \subset p(U), q\left(z_{0}\right)=p\left(\zeta_{0}\right)$, and

i) $D q\left(z_{0}\right)=m D p\left(\zeta_{0}\right)$;

ii) $\operatorname{Re} \frac{D^{2} q\left(z_{0}\right)}{D q\left(z_{0}\right)} \geq m \operatorname{Re} \frac{D^{2} p\left(\zeta_{0}\right)}{D p\left(\zeta_{0}\right)}$.

\section{Main results}

In paper [9], S.S. Miller and P.T. Mocanu have introduced the dual notion of the differential superordination for analytic functions. In this paper we extend this notion for the harmonic complex-valued functions following the classical theory of differential superordination.

Definition 2.1. Let $f$ and $F$ be members of $\operatorname{Har}(U)$. The function $f$ is said to be subordinate to $F$, or $F$ is said to be superordinate to $f$, if there exist a function $w$ analytic in $U$, with $w(0)=0$ and $|w(z)|<1$ such that $f(z)=F(w(z))$. In such a case we write $f(z) \prec F(z)$. If $F$ is univalent in $U$, then $f(z) \prec F(z)$ if and only if $f(0)=F(0)$ and $f(U) \subset F(U)$.

Let $\Omega$ and $\Delta$ be any sets in $\mathbb{C}$, let $p$ be a harmonic complex-valued function in the unit disc $U$ and let $\varphi(r, s, t ; z): \mathbb{C}^{3} \times U \rightarrow \mathbb{C}$. In this paper we consider the problem of determining conditions on $\Omega, \Delta$ and $\varphi$ for which the following implication holds:

$$
\Omega \subset\left\{\varphi\left(p(z), D p(z), D^{2} p(z) ; z\right): z \in U\right\} \Rightarrow \Delta \subset p(U) .
$$

There are three distinct cases to consider in analyzing this implication, which we list as the following problems:

Problem 1. Given $\Omega$ and $\Delta$, find conditions on the function $\varphi$ so that (2.1) holds.

Problem 2. Given $\varphi$ and $\Omega$, find a set $\Delta$ such that (2.1) holds.

Furthermore, find the largest such $\Delta$.

Problem 3. Given $\varphi$ and $\Delta$, find a set $\Omega$, such that (2.1) holds.

Furthermore, find the smallest such $\Omega$.

If either $\Omega$ or $\Delta$ in (2.1) is a simply connected domain, then it may be possible to rephrase (2.1) in terms of superordination. If $p$ is harmonic univalent in $U$, and if $\Delta$ is a simply connected domain with $\Delta \neq \mathbb{C}$, then there is $g$ a harmonic and univalent function, conformal mapping of $U$ onto $\Delta$, such that $q(0)=p(0)$.

In this case (2.1) can be rewritten as

$$
\Omega \subset\left\{\varphi\left(p(z), D p(z), D^{2} p(z) ; z\right)\right\} \Rightarrow q(z) \prec p(z) .
$$

If $\Omega$ is also a simply connected domain with $\Omega \neq \mathbb{C}$, then there is a conformal mapping $h$ of $U$ onto $\Omega$, harmonic univalent function such that $h(0)=\varphi(p(0), 0,0 ; 0)$. 
If in addition, the function $\varphi\left(p(z), D p(z), D^{2} p(z) ; z\right)$ is harmonic univalent in $U$, then (2.1) can be rewritten as

$$
h(z) \prec \varphi\left(p(z), D p(z), D^{2} p(z) ; z\right) \Rightarrow q(z) \prec p(z), z \in U .
$$

In the special case when the set inclusion (2.1) can be replaced by the superordination for harmonic complex-valued function (2.2), we can reinterpret the three problems referred to above as follows:

Problem 1'. Given harmonic complex-valued functions $h$ and $q$, find a class of admissible functions $\Phi[h, q]$ such that (2.2) holds.

Problem 2'. Given the differential superordination for harmonic complex-valued functions (2.2), find a subordinant $q$. Moreover, find the best subordinant.

Problem 3'. Given $\varphi$ and subordinant $q$, find the largest class of harmonic complexvalued functions $h$ such that (2.2) holds.

Remark 2.1. A function $f(z)=a \bar{z}+b, a \neq 0, a, b \in \mathbb{C}$, which is a harmonic function, is a conformal mapping of the complex plane into itself. we let

Let $z=x+i y, \bar{z}=x-i y, a=a_{1}+i a_{2}, a_{1} \neq 0$ or $a_{2} \neq 0, b=b_{1}+i b_{2}$. Then

$$
f(z)=a_{1} x+a_{2} y+b_{1}+i\left(a_{2} x-a_{1} y+b_{2}\right)
$$

Denote by

$$
P(x, y)=a_{1} x+a_{2} y+b_{1}, Q(x, y)=a_{2} x-a_{1} y+b_{2} .
$$

The functions $P$ and $Q$ are continuous functions which admit partial derivatives with respect to $x$ and $y$. We have

$$
\frac{\partial P(x, y)}{\partial x}=a_{1}, \frac{\partial Q(x, y)}{\partial y}=-a_{1}, \frac{\partial P(x, y)}{\partial y}=a_{2}, \frac{\partial Q(x, y)}{\partial x}=a_{2}
$$

Since

$$
\frac{\partial^{2} P(x, y)}{\partial x^{2}}+\frac{\partial^{2} P(x, y)}{\partial y^{2}}=0 \text { and } \frac{\partial^{2} Q(x, y)}{\partial x^{2}}+\frac{\partial^{2} Q(x, y)}{\partial y^{2}}=0
$$

we get that the function $f$ is a harmonic function.

We now show that function $f$ is a conformal mapping.

Let $a=|a| e^{i \phi},|a|=R>0, \phi=\arg a, \bar{z}=|\bar{z}| e^{i \theta}=|z| e^{i \theta}, \theta=\arg \bar{z}$.

Then $f(z)=R e^{i \phi} \bar{z}+b$ can be decomposed into three elementary substitutions:

(1) $z_{1}=e^{i \phi} \bar{z}=|z| e^{i(\theta+\phi)}$, meaning that the point $z_{1}$ can be obtained by the rotation of the entire complex plane around the origin by a constant angle $\phi$. Rotation preserves the angles of the rotated figures.

(2) $z_{2}=R z_{1}$, where $R>0$, and a constant. This is a homothetic transformation. It is well-known that the homothetic transformation only changes the dimensions of the figures without changing the shape and it preserves the angles.

(3) $w=z_{2}+b$, which is a translation of the complex-plane, characterized by $b$. Translation preserves dimensions and shape, hence it preserves the angles.

Since $f(z)=a \bar{z}+b$, is a combination between a rotation, a homothetic transformation and a translation, $f$ preserves angles, hence it is a conformal mapping. 
Definition 2.2. Let $\varphi: \mathbb{C}^{3} \times U \rightarrow \mathbb{C}$ and let $h$ be harmonic univalent in $U$. If $p$ and $\varphi\left(p(z), D p(z), D^{2} p(z)\right)$ are harmonic univalent in $U$, and satisfy the second-order differential superordination for harmonic complex-valued functions

$$
h(z) \prec \varphi\left(p(z), D p(z), D^{2} p(z) ; z\right)
$$

then $p$ is called a solution of the differential superordination.

A harmonic univalent function $q$ is called a subordinant of the solutions of the differential superordination for harmonic complex-valued functions, or more simply a subordinant if $q \prec p$, for all $p$ satisfying (2.3). An univalent harmonic subordinant $\bar{q}$ that satisfies $q \prec \bar{q}$ for all subordinants $q$ of (2.3) is said to be the best subordinant. The best subordinant is unique up to a rotation of $U$.

Remark 2.2. For $\Omega$ a set in $\mathbb{C}$, with $\varphi$ and $p$ as given in Definition 2.2, suppose (2.3) is replaced by

$$
\Omega \subset\left\{\varphi\left(p(z), D p(z), D^{2} p(z): z \in U\right\} .\right.
$$

Although this more general situation is a differential containment, the condition in (2.4) will also be referred to as a differential superordination for harmonic complexvalued functions, and the definitions of solution, subordinant and best subordinant as given above can be extended to this generalization.

We next give the definition of the class of admissible function for harmonic complex-valued functions.

Definition 2.3. Let $\Omega$ be a set in $\mathbb{C}$ and let $q$ be a harmonic univalent function. The class of admissible functions $\Phi[\Omega, q]$ consists of those functions $\varphi: \mathbb{C}^{3} \times \bar{U} \rightarrow \mathbb{C}$ that satisfy the admissibility condition

$$
\varphi(r, s, t ; \zeta) \in \Omega
$$

where

$$
r=q(z), s=\frac{D q(z)}{m}, \operatorname{Re}\left(\frac{t}{s}+1\right) \leq \frac{1}{m} \operatorname{Re} \frac{D^{2} q(z)}{D q(z)},
$$

where $\zeta \in \partial U, z \in U$ and $m \geq 1$.

If $\varphi: \mathbb{C}^{2} \times \bar{U} \rightarrow \mathbb{C}$, the admissibility condition $(A)$ reduces to

$$
\varphi\left(q(z), \frac{D q(z)}{m} ; \zeta\right) \in \Omega
$$

where $z \in U, \zeta \in \partial U$ and $m \geq 1$.

In the special case when $h$ is a harmonic complex-valued function conformal mapping of $U$ onto $\Omega \neq \mathbb{C}$, we denote the class $\Phi[h(U), q]$ by $\Phi[h, q]$.

The following theorems are important results for the theory of differential superordinations for complex-valued harmonic functions.

Theorem 2.1. Let $\Omega \subset \mathbb{C}$, let $q$ be a harmonic and univalent function with $q(0)=$ 1 and let $\varphi \in \Phi[\Omega, q]$. If $p \in Q, p(0)=1, p(U)$ is simply connected and $\varphi\left(p(z), D p(z), D^{2} p(z): z \in U\right)$ is harmonic and univalent in $U$, then

$$
\Omega \subset\left\{\varphi\left(p(z), D p(z), D^{2} p(z): z \in U\right)\right\}
$$


implies

$$
q(z) \prec p(z), z \in U .
$$

Proof. Assume $q \nprec p$. From Lemma 1.1, there exist points

$$
z_{0}=r_{0} e^{i \theta_{0}} \in U \text { and } \zeta_{0} \in \partial U \backslash E(q), m \geq 1,
$$

that satisfy

$$
q\left(z_{0}\right)=p\left(\zeta_{0}\right), D q\left(z_{0}\right)=m D p\left(z_{0}\right), \operatorname{Re} \frac{D^{2} q\left(z_{0}\right)}{q\left(z_{0}\right)} \geq m \operatorname{Re} \frac{D^{2} p\left(\zeta_{0}\right)}{D p\left(\zeta_{0}\right)} .
$$

Let $r=p\left(\zeta_{0}\right), s=D p\left(\zeta_{0}\right), t=D^{2} p\left(\zeta_{0}\right)$, and $\zeta=\zeta_{0}$, in Definition 2.3, then we obtain

$$
\varphi\left(p\left(\zeta_{0}\right), D p\left(\zeta_{0}\right), D^{2} p\left(\zeta_{0}\right) ; \zeta_{0}\right) \in \Omega
$$

Since this contradicts (2.5), we have that the assumption made is false, hence $q(z) \prec p(z), z \in U$.

Remark 2.3. If $h$ is a harmonic and univalent function in $U$, is a conformal mapping and $h(U)=\Omega \neq \mathbb{C}$, then the class $\Phi[h(U), q]$ is written as $\Phi[h, q]$ and the following result is an immediate consequence of Theorem 2.1.

Theorem 2.2. Let $q$ be a harmonic and univalent function in $U$, with $q(0)=1$, let $h$ be harmonic and univalent in $U$, with $p(0)=1, p(U)$ is simply connected and $\varphi \in \Phi[h(U), q]$. If $p \in Q$ and $\varphi\left(p(z), D p(z), D^{2} p(z) ; z\right)$ is harmonic and univalent in $U$, then

$$
h(z) \prec \varphi\left(p(z), D p(z), D^{2} p(z) ; z\right)
$$

implies

$$
q(z) \prec p(z), z \in U .
$$

From Theorem 2.1 and Theorem 2.2, we see that we can obtain subordinants of a differential superordination for harmonic complex-valued functions of the form (2.5) and (2.6), by simply checking that the function $\varphi$ is an admissible function.

The following theorem proves the existence of the best subordinant of (2.6) for certain $\varphi$ and also provides a method for finding the best subordinant.

Theorem 2.3. Let $h$ be a harmonic and univalent function in $U, h(U)$ is simply connected and let $\varphi: \mathbb{C}^{3} \times \bar{U} \rightarrow \mathbb{C}$. Suppose that the differential equation

$$
\varphi\left(q(z), D q(z), D^{2} q(z) ; z\right)=h(z)
$$

has a solution $q \in Q$, harmonic and univalent in $U$. If $\varphi \in \Phi[h(U), q], p \in Q, p(0)=1$, $p(U)$ is simply connected and $\varphi\left(p(z), D p(z), D^{2} p(z) ; z\right)$ is harmonic and univalent in $U$, then

$$
h(z) \prec \varphi\left(p(z), D p(z), D^{2} p(z) ; z\right)
$$

implies

$$
q(z) \prec p(z), z \in U,
$$

and $q$ is the best subordinant. 
Proof. Since $\varphi \in \Phi[h(U), q]$ and is harmonic and univalent in $U$, by applying Theorem 2.2 , we deduce that $q$ is a subordinant of (2.8). Since $q$ also satisfies (2.7), it is also a solution of the differential subordination (2.8) and therefore all subordinants of (2.8) will be subordinate to $q$. Hence $q$ will be the best subordinant of (2.8).

From this theorem we see that the problem of finding the best subordinant of (2.8) essentially reduces to showing that differential equation (2.8) has an univalent solution and checking that $\varphi \in \Phi[h(U), q]$.

\section{First-order differential superordinations for harmonic complex-valued functions}

We can simplify Theorem 2.1, 2.2 and 2.3 for the case of first-order differential superordinations for harmonic complex-valued functions.

The following results are immediately obtained by using these theorems and admissibility condition $\left(A^{\prime}\right)$.

Theorem 3.1. Let $\Omega \subset \mathbb{C}$, let $q$ be a harmonic and univalent function with $q(0)=1$ and $\varphi \in \Phi[\Omega, q]$. If $p \in Q$ and $\varphi(p(z), D p(z) ; z \in U)$ is harmonic and univalent in $U$, then

$$
\varphi(q(z), t D q(z) ; \zeta) \in \Omega
$$

for $z \in U, \zeta \in \partial U$ and $0<t \leq \frac{1}{m} \leq 1, m \geq 1$. If $p \in Q, p(0)=1, p(U)$ is simply connected and $\varphi(p(z), D p(z) ; z)$ is harmonic and is univalent in $U$, then

$$
\Omega \subset\{\varphi(p(z), D p(z)) ; z \in U\}
$$

implies

$$
q(z) \prec p(z), z \in U \text {. }
$$

Theorem 3.2. Let $h, q$ be harmonic and univalent functions in $U, \varphi: \mathbb{C}^{2} \times \bar{U} \rightarrow \mathbb{C}$, and suppose that

$$
\varphi(q(z), t D q(z) ; \zeta) \in h(U)
$$

for $z \in U, \zeta \in \partial U$ and $0<t \leq \frac{1}{m} \leq 1, m \geq 1$. If $p \in Q, p(0)=1, p(U)$ is simply connected and $\varphi(p(z), D p(z) ; z \in U)$ is harmonic and univalent in $U$, then

$$
h(z) \prec \varphi(p(z), D p(z) ; z)
$$

implies

$$
q(z) \prec p(z), z \in U .
$$

Furthermore, if $\varphi(q(z), D q(z) ; z)=h(z)$, has a univalent solution $q \in Q$, then $q$ is the best subordinant.

We next give an example of finding the best subordinant of a differential superordination of harmonic functions. 
Example 3.1. Let $q(z)=1+M z+\bar{z}, z \in U, M>0$ be a harmonic complex-valued function in the unit disc.

Let $z \in U, z=x+i y, \bar{z}=x-i y$.

Then

$$
q(z)=1+x+M x+i(M y-y)
$$

We denote

$$
P(x, y)=1+x+M x, Q(x, y)=M y-y .
$$

The functions $P$ and $Q$ are continuous functions in $U$ which admit partial derivatives with respect to $x$ and $y$. We have

$$
\frac{\partial P(x, y)}{\partial x}=1+M, \frac{\partial P(x, y)}{\partial y}=0, \frac{\partial Q(x, y)}{\partial x}=0, \frac{\partial Q(x, y)}{\partial y}=M-1
$$

Since $\frac{\partial^{2} P(x, y)}{\partial x^{2}}+\frac{\partial^{2} P(x, y)}{\partial y^{2}}=0$ we have that $P(x, y)$ is a harmonic function.

Since $\frac{\partial^{2} Q(x, y)}{\partial x^{2}}+\frac{\partial^{2} Q(x, y)}{\partial y^{2}}=0$ we have that $Q(x, y)$ is a harmonic function.

Hence, $f(z)=P(x, y)+i Q(x, y)$ is a harmonic function.

The function $q(z)=1+M z+\bar{z}$ is the univalent harmonic solution of the equation

$$
h(z)=q(z)+D q(z)+D^{2} q(z)=1+3 M z+\bar{z}
$$

which is an univalent harmonic function.

If

$$
1+3 M z+\bar{z} \prec p(z)+D p(z)+D^{2} p(z),
$$

then, using Theorem 2.3, we have that

$$
1+M z+\bar{z} \prec p(z), z \in U
$$

and $q(z)=1+M z+\bar{z}$ is the best subordinant.

\section{References}

[1] Bulboacă, T., Differential Subordinations and Superordinations. Recent Results, Casa Cărţii de Ştiinţă, Cluj-Napoca, 2005.

[2] Clunie, J.G., Sheil-Small, T., Harmonic univalent functions, Ann. Acad. Sci. Fenn. Math., 9(1984), 3-25.

[3] Duren, P.L., Harmonic Mappings in the Plane, Cambridge Tracts in Mathematics, 156, Cambridge Univ. Press, 2004.

[4] Lewy, H., On the non-vanishing of the Jacobian in certain one-to-one mappings, Bull. Amer. Math. Soc., 42(1936), 689-692.

[5] Kanas, S., Differential subordinations for harmonic complex-valued functions, arxiv: 1509.03751V1 [math. CV], 12 sep. 2015.

[6] Miller, S.S., Mocanu, P.T., Second order differential inequalities in the complex plane, J. Math. Anal. Appl., 65(1978), 298-305.

[7] Miller, S.S., Mocanu, P.T., Differential subordinations and univalent functions, Michig. Math. J., 28(1981), 157-171. 
[8] Miller, S.S., Mocanu, P.T., Differential Subordinations, Theory and Applications, Marcel Dekker Inc., New York, Basel, 2000.

[9] Miller, S.S., Mocanu, P.T., Subordinants of differential superordinations, Complex Variables, 48(10)(2003), 815-826.

[10] Mocanu, P.T., Bulboacă, T., Sălăgean, G.Şt., Geometric Function Theory, (Romanian), Casa Cărţii de Ştiinţă, Cluj-Napoca, 1999.

[11] Oros, G.I., Using Differential Subordinations in the Study of Certain Classes of Univalent Functions, (Romanian), Casa Cărţii de Ştiinţă, Cluj-Napoca, 2008, 208 pp.

[12] Oros, G.I., New Differential Subordinations and Superordinations, Lambert Academic Publishing, Saarbrucken, Germany, 2011, 263 pp.

[13] Oros, Gh., Convexity and Starlikeness in Geometric Function Theory, Caretaken by the PAMM, Centre at the Bute, Budapest, 2001.

[14] Schaubroech, L., Subordination of planar harmonic functions, Complex Var. Theory Appl., 41(2000), 163-178.

Georgia Irina Oros

Department of Mathematics, University of Oradea Str. Universităţii, No. 1, 410087 Oradea, Romania

e-mail: georgia_oros_ro@yahoo.co.uk

Gheorghe Oros

Department of Mathematics, University of Oradea

Str. Universităţii, No. 1, 410087 Oradea, Romania 